\title{
LA RESTAURATION DES PEINTURES MURALES D'ÉPOQUE ROMAINE
}

\author{
par Alix BARBET
}

La France possède de nombreux sites archéologiques ayant livé des fragments de peinture murale* romaine mais cette source de renseignements sur la vie artistique et les modes de décor de cette époque sont assez peu exploités, faute d'une politique de restauration ${ }^{1}$. Les fragments exhumés restent à l'état de fragments et, entassés dans les réserves des musées, achèvent trop souvent de se désagréger. Quand, par hasard, le fouilleur tente une restauration, laissée à sa propre initiative, les résultats sont fréquemment médiocres, quand ils ne sont pas désastreux à cause de l'emploi, sans expérimentation préalable en laboratoire, de toutes sortes de produits nocifs. Quant aux peintures restant en place, sur les murs qu'elles lécoraient, les intempéries et le soleil ont vite fait de les oblitérer.

Or, lorsqu'on se penche sur ces débris, parfois bien médiocres, on est largement récompensé de ses efforts, car, après un patient travail de reconstitution* et de restauration, il n'est pas rare d'obtenir des résultats extraordinaires. L'intérêt de la peinture murale romaine pour la compréhension d'un site et la datation d'un édifice, est alors d'une grande importance : une bonne reconstitution peut donner les dimensions approximatives d'un mur ruiné, notamment sa hauteur, et, si son style est caractéristique, elle fournira des éléments de chronologie appréciables. Il est inutile de souligner qu'une étude sérieuse de peintures murales exige une restauration préalable des fragments peints à étudier.

Les principales difficultés que rencontrent presque tous les fouilleurs de sites romains sont nombreuses et commencent dès l'instant où il faut récolter les fragments de peinture. Nous nous attacherons à proposer différentes solutions, en exposant plusicurs méthodes, en faisant part de nos propres expériences, encore très limitées, sur des peintures romaines trouvées en Gaule, mais il est bien entendu que chaque cas est particulier et qu'il faut toujours lui adapter la solution choisie, pour opérer une restauration. Avant tout traitement, une peinture murale doit être examinée pour déterminer les causes d'altération et y remé-

1 Les mots suivis d'un astérisque figurent dans le grossaire des termes techniques ou dans le calalogue des produits chimiques, ci-dessous, p. 89-92. 
dier. I'après P. Philippot et P. Mora ${ }^{2}$, voici quelles sont les données à relever, surtout lorsqu'il s'agit d'une peinture en place :

1) nature et état du mur ou de la structure qui supporte la peinture, en vue de déterminer les sources possibles d'humidilé dues à la capillarité, la condensalion ou l'infiltration ;

2) stabilité du mur ;

3) sondages en vue de déterminer le degré de cohésion des couches d'enduit* et de morlier* de leur adhérence entre elles et au mur;

4) relevé et identification des altérations et détermination de leurs causes;

5) étude de la couche picturale pour en déterminer la technique et les matériaux constitutifs, composition des enduits, identification des pigments*, des liants*, éventuellement des restaurations et des couches superposées après l'achèvement de l'œuvre, identification de sels, de micro-organismes, elc. ;

6) relevé et étude de tous les éléments permettant de préciser la chronologie du travail. On peut y joindre une recherche bibliographique et constituer une documentation photographique.

Ln des points délicats est la détermination de la technique de peinture utilisée. Ceci est fort important. Par exemple, si l'on identifie une détrempe soluble dans l'eau, cela élimine d'office certaines méthodes de restauration. I)ans la plupart des cas, la détermination exacte des techniques picturales anciennes est malaisée. Mème l'identification de la fresque ${ }^{*}$ pure est délicate, comme le souligne très bien un passage important du rapport de P. Philippot et P. Mora ${ }^{3}$; c'est pourquoi il faut éviter de parler de "fresques" et se contenter du terme générique de "peintures murales ». En effet, la seule présence de carbonate de chaux entre les pigments n'est pas décisive car, si celui-ci peut provenir de la carbonatation de la chaux* dans la réaction de la fresque, il peut aussi avoir été appliqué comme pigment. Il faudrait donc, pour pouvoir conclure à la présence de fresque, être en mesure d'exclure l'intervention de tout liant de détrempe, ce qui sera généralement difficile, car il peut ne laisser que des traces infimes.

Pour les peintures de Glanum, nous avons fait faire des analyses par le laboratoire du Louvre el celui de l'Islituto Centrale del Restauro de Rome* ${ }^{4}$ on a pu mettre en évidence une exécution de fresque mixte*, avec emploi d'un liant de nature protéique. Ainsi les cas ne sont pas simples, malgré l'utilisation d'analyses micro-chimiques et stratigraphiques des coupes, de photographies aux rayons infra-rouges, elc.

Pour examiner les altérations, outre l'examen à l'œil nu qui permet de déceler déjà s'il s'agit de saletés à la surface, de lacunes, d'efflorescences, d'incrustations, l'examen à la lumière rasante conduit au contrôle de l'adhérence de la couleur. Pour examiner l'adhérence de l'enduit au support, on peut tapoter la surface avec un doigt : si les coups rendent un son creux, c'est signe qu'une poche d'air s'est formée entre l'enduit et le support ; on relève

2 P. PHIIPpoT, P. Mora, Rapport sur les peintures murales, ICO.M, 1965. ex. dactyl., p. III, 5. Ce maport tries riche nous a fourni une mine de renseignements, atondamment ulilises ici. Je remercie le professeur .lora qui a e"l l'amabilité de m'en prèter un exemplaire.

3 P. PHII.PPOT, P. HORA, op. cil., p. III, 6-7.

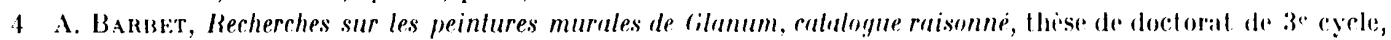
non publiép, p. 77 it s. 
alors exactement l'emplacement de ces manques d'adhérence pour savoir, lors de la restauration, où injecter du caséate de chaux. Lorsque la peinture, fort usée, est devenue d'une lecture difficile, on a avantage à la photographier sous éclairage fluorescent, ce qui augmente la lisibilité.

Cies examens, indispensables lorsqu'on trouve une peinture en place, sont très réduits lorsqu'il s'agit de morceaux tombés au bas d'un mur. Il suffit alors de procéder à un ramassage et à un nettoyage méticuleux.

\section{Ramassage et traitement immédiat des fragments de peintures tombés}

Ln certain nombre de précautions sont à prendre : il faut, cela va sans dire, ramasser lous les fragments, même ceux qui ne portent pas de décor, y compris ceux qui sont d'un blanc uni, attendu que lors de la reconstitution, chaque fragment est utile. Un quadrillage méthodique aura été établi et reporté sur les caisses. La réussite de la reconstitution dépend de la minutic de ces opérations. Il ne faut pas entasser les débris sans séparer les différentes couches au moyen de vétroflex ou, au moins, de papier journal, pour éviter les rayures sur les surfaces picturales ${ }^{5}$.

Ces détails importent beaucoup car nous connaissons des cas où une partie des collections de peintures murales d'un musée a été irrémédiablement perdue pour la science, faute de ces simples précautions. Il ne faut pas non plus laisser les fragments sortis de fouille à l'air libre, pour qu'ils sèchent, car, avec l'humidité, l'air et le soleil sont les pires ennemis des enduits. Enfin, nous déconseillons également de dissocier les couches de mortier supportant la peinture, de supprimer la dernière, plus friable : cela est désastreux pour la recherche des morceaux qui recollent entre eux et l'étude technique des composants, sans parler de la fragilité accrue des fragments ainsi émincés.

Après avoir attendu un séchage parfait des peintures humidifiées par la terre, on peut procéder au nettoyage, qui se fera de préférence avec une éponge mouillée, lorsqu'on aura reconnu qu'on n'a pas affaire à une détrempe. On peut ensuite trier les couleurs, faire les collages (fig. 5), recomposer de proche en proche le décor originel (fig. 6). Selon les cas, on arrivera à une reconstitution très complète, ou seulement à une restitution *, du décor à l'aide de reconstitutions partielles. En attendant la mise sur panneaux, il convient d'entreposer l'ensemble sur du sable, à l'abri du froid autant que possible, mais surtout de l'humidité, du soleil et des changements brusques de température (fig. 7). Il importe que le degré hygrométrique soit à peu près constant. La tendance actuelle vise à laisser la surface libre sans la protéger par un fixatif, sauf cas de nécessité absolue. On peut alors utiliser de la gomme-laque* blanche en poudre, diluée dans de l'alcool, ou d'autres substances signalées ci-dessous (II, b).

5 Cf. autres conseils pratiques : A. Barbet, La peinlure romaine, Nolice technique du Touring-Chuh de France, Paris, 1969. 


\section{Protection d'une peinture murale en place}

Il n'existe aucun moyen absolument satisfaisant à l'heure actuelle pour maintenir en place une peinture murale sans qu'elle s'abîme, du moins sous nos climats. Ceux qui ont tenté l'expérience ont pu s'apercevoir que la peinture pâlit considérablement, malgré les abris construits à cet effet, et qu'au bout de peu de temps les motifs s'effacent. Parfois c'est le support qui ne résiste pas et le mur peint tombe par plaques, peu à peu.

Pour illustrer ces considérations, qui peuvent paraitre pessimistes, voici deux exemples pris dans le Midi de la France. Après sept ans de mise au jour, une peinture appliquée sur mur d'argile séchée était réduite aux deux tiers (fig. 1 et 2). Aujourd'hui, au bout de dix ans, il n'en reste quasiment. rien. Quant à une très belle peinture, restaurée et replacée à l'endroit d'origine, ses couleurs ont. passé et elle pâlit de plus en plus, jusqu'au jour où on ne distinguera plus rien (fig. 3 et 4 ). Bien entendu, nous ne mettons pas en cause le directeur du chantier de fouilles, qui, en ce qui concerne la remise en place de la peinture restaurée, n'a fait que suivre les conseils d'un restaurateur.

Nous insistons donc sur la nécessité en France, où le climat est humide, de déposer toutes les peintures murales romaines, mème celles dont le support et la pellicule picturales sont en excellent état car, à plus ou moins brève échéance, elles sont promises à la disparition. Il est toujours fâcheux de dissocier l'architecture de son décor, mais nous pensons néanmoins qu'il est préférable de ne pas replacer dans son lieu d'origine un panneau de peinture déposé et restauré convenablement : il faut le mettre à l'abri dans un musée ou un dépôt, quitte à reconstituer une salle peinte entière, si les éléments le permettent. Les Italiens eux-mèmes, partisans, en général, du replacement des panneaux restaurés en leur lieu d'origine, moyennant les précautions nécessaires, commencent à abriter dans leurs musées certaines peintures, par exemple celles de la villa de Livie à Prima Porta (au Musée national des Thermes), une partie de celles de la maison des (Griffons sur le Palatin (au Musée du Palatin). La solution provisoire, lorsqu'on ne peut faire détacher la peinture immédiatement, est de remblayer la fouille à cet endroit avec du sable, après avoir procédé à des relevés graphiques et photographiques très complets, à un nettoyage, à un fixage très soigneux des peintures et à une consolidation si nécessaire ${ }^{6}$.

a) Netloyage. En cas de matières grasses, provenant notamment de fumées ou de colles animales appliquées au cours de restaurations antérieures, il faut recourir à des solvants en commençant par les plus faibles?. Dans la plupart des cas, une solution d'eau avec $10 \%$ d'ammoniaque $(20 \%$ au maximum) suffit : on l'applique doucement avec un tampon d'ouate en tamponnant prudemment sur les couleurs délicates ${ }^{8}$. Pour enlever les repeints à l'huile lorsqu'il y a eu restauration abusive, on utilisera par ordre de force croissante, la

6 Dans son ouvrage Die Kömische Wandmalerei der schueiz, Bâle, 1950, p. 4, 11. Drack ast sensible à ce prohleme de conservation des peintures murales et dit avoir remblayé une fouille a Hejggel pour protéger des peintures, ne sachant pas comment faire autrement, lorsqu'il était jeune archéologue.

7 1.. V. Borreit.ı, Restauro, dans Enciclopedia universale dell'arte, XI, Rome-Venise, 1963, p. 338.

8 P. Phillppot, P. MoRA, op. cil., p. III, 10-12. 


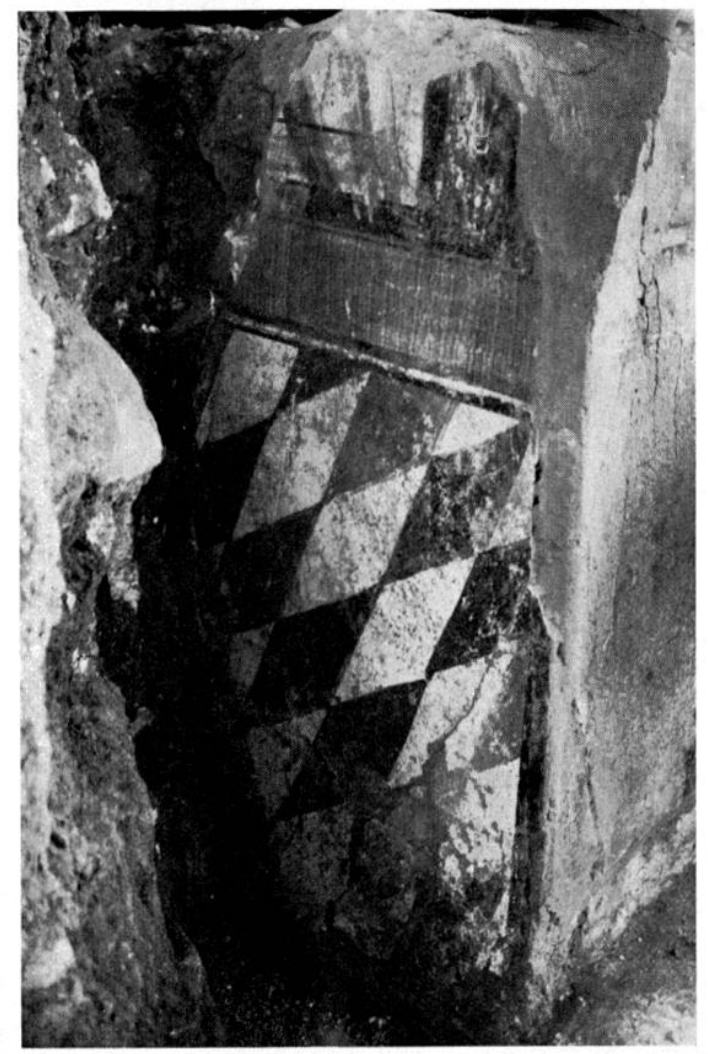

1 Peinture murale, all moment de lat découterte, posée sur un mur dargile sechere.

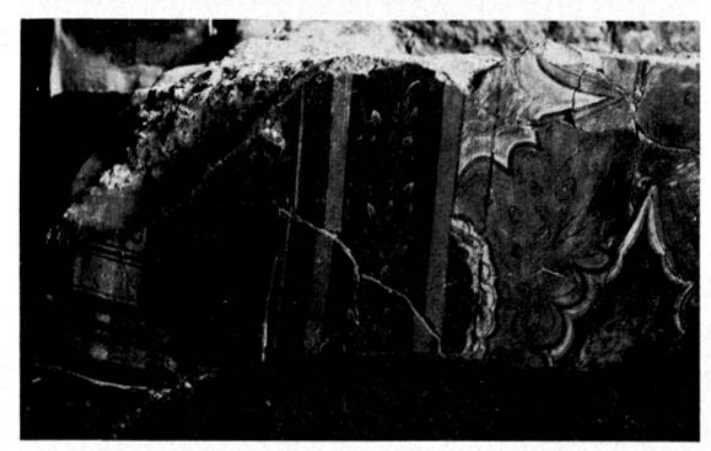

3 Peinture murale, lors de la découverte : les couleurs sonl tres vives.

4 La mème peinture, sept ans plus lard : malgré $\rightarrow$ la restauration ef la mise sur un mur sain, la peinture se degrade dans son milien diorigine.

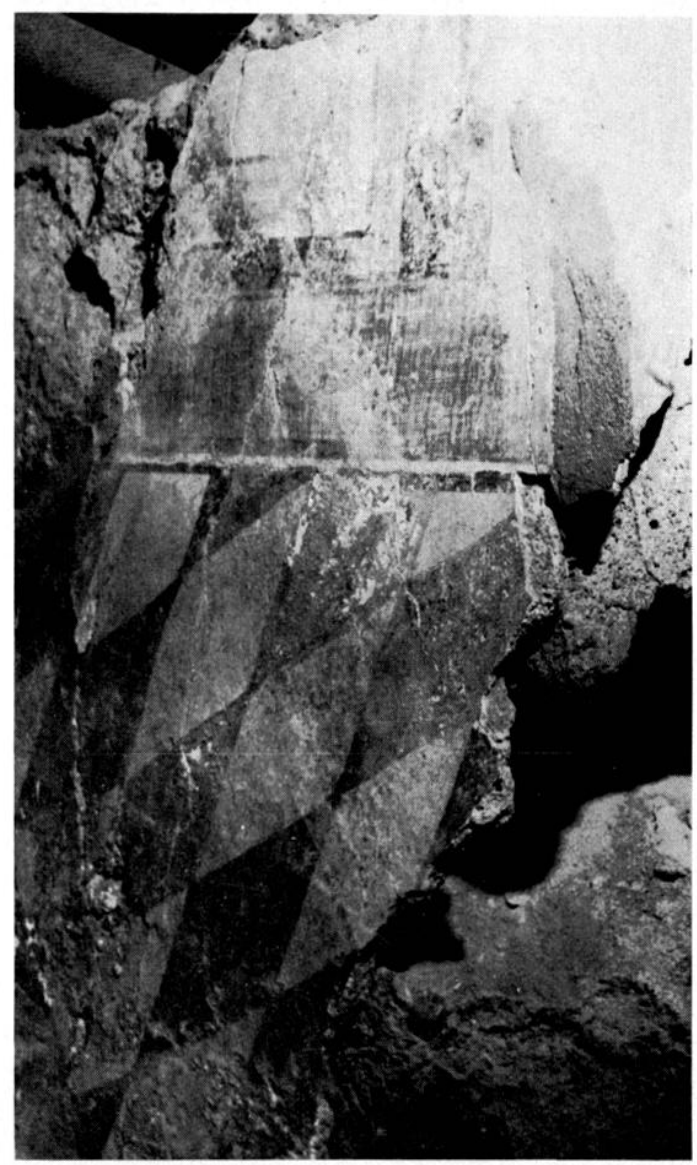

2 La même peinture, sept ans plus tard : l'argile s'est réduite en poudre, la peinture, déjà amputér d'un tiers de son decor, est dans le vide.

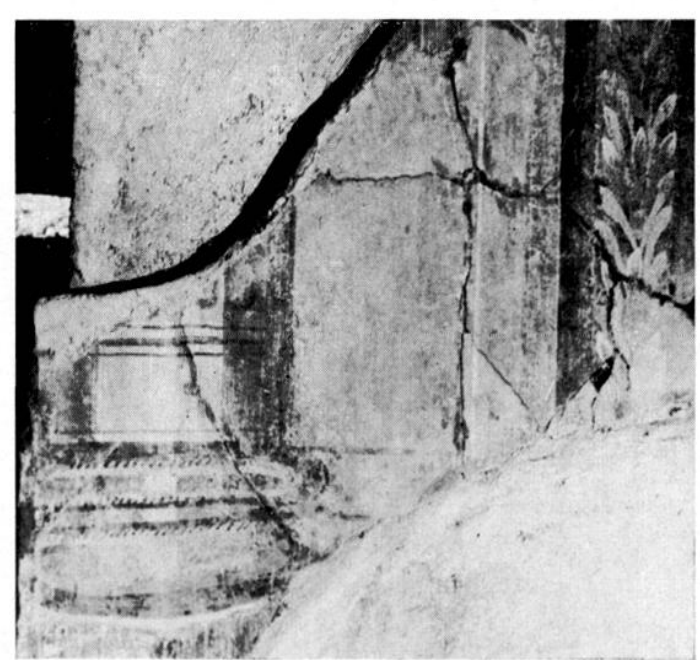


butilamine (10) \% à $20 \%$ dans de l'eau), la cycloexylamine $(80 \%$ à $90 \%$ dans de l'eau). Pour enlever la cire, dont on a longtemps cru bon d'enduire les peintures, spécialement celles qui sont conservées dans les musées et qui subissent un assombrissement et un encrassement prononcés, on doit utiliser le tétrachlorure de carbone ou le trichlorétylène. Pour les efflorescences salines, un lavage à l'eau est nécessaire, suivi d'une application de papier humide pour les dissoudre et les attirer à la surface, où elles se cristallisent. Pour les sels insolubles, les dépôts organiques et les badigeons ultérieurs, si fréquents, seul le traitement mécanique est praticable : avec un grattoir et une loupe, il faut patiemment enlever les dépôts (prudemment, pour ne pas entamer la couleur) et terminer par un lavage à l'eau avec $10 \%$ à $20 \%$ d'ammoniaque.

Contre le développement des végétaux et des moisissures, qui se produit à la faveur d'une humidité relative trop élevée (au-dessus de $70 \%$ pour les moisissures), il faut à la fois réduire l'excès de l'humidité -.. les Allemands emploient pour assécher un mur des lampes à infra-rouges (chaque lampe, $3 \mathrm{kw} / \mathrm{h}$ ) —9 et utiliser un agent stérilisant. Actuellement, le fongicide à recommander est la formaline, appliquée au pinceau, ou par vaporisation. Son inconvénient est de rendre insolubles les colles animales, employées fréquemment lors des opérations de dépose. On peut prendre un produit plus puissant, le pentachlorophénate de sodium en solution aqueuse à $2 \%$, qui est à appliquer avec une brosse douce. La protection est alors durable.

b) Fixalion des couleurs. Après avoir nettoyé les peintures, il convient parfois de les fixer, surtout lorsque l'enduit perd sa cohésion et devient pulvérulent. La définition du bon fixatif a été excellemment donnée dans le rapport déjà cité $e^{10}$ : il doit avoir un pouvoir adhésif suffisant, bien pénétrer dans la surface traitée, résister à l'abrasion et aux microorganismes, être flexible et rester soluble. Il doit aussi être incolore, ne pas modifier les couleurs, la texture de la peinture. Enfin, il doit conserver toutes ces qualités avec le temps. C'est dans ce domaine, en général, que les non-spécialistes appliquent des produits sans savoir qu'ils sont nocifs; nous en donnons une liste, non exhaustive, pour les inviter à plus de prudence :

les silicates inorganiques peuvent produire des efflorescences, les silicates organiques ne restent pas adhésifs ; les silicones peuvent, théoriquement, former une pellicule de silice d'un blanc laiteux et opaque ; les cires et les cires-résines imperméabilisent en surface la peinture, qui peut rester humide en profondeur, favorisent l'encrassement et peut-être même le développement des micro-organismes. La seule règle est la prudence dans l'expérimentation, sous le contròle de services compétents mais ceux-ci n'existent pas encore en France, d'où les ravages que nous pouvons constater sur des documents définitivement endommagés par des traitements fantaisistes.

Les prorluits retenus par l'Institut de la Restauration de Rome sont le Paraloid B 72*, ou Acryloid B 72 (acrylate de méthyle et métacrylate d'éthyle en solution à $5 \%$ dans le toluène), le Bedacryl 122. Il faut travailler dans des locaux bien aérés car ces produits sont

9 K. Wins, Bergung und Konservierung der rämischen Wandmalereien aus der Trierer Kaiserlhermengrabung $19 t 22$, dans Trierer Zeilschrift, 29, 1966, p. 224.

10 P. P'HIIPPOT, J'. MORA, op. cil., p. II, 1.4 ets. 
toxiques. Quant à la gomme-laque, utilisée depuis longtemps, c'est un excellent adhésif, employé même comme colle pour détacher des peintures très humides comme celles des tombeaux étrusques ${ }^{11}$, mais elle jaunit avec le temps et devient insoluble. Il vaut mieux donc ne l'utiliser que rarement.

c) Consolidalion de l'enduil el du mortier. Ceci est particulièrement utile lorsqu'un mur peint se fissure et devient ventru. Plutôt que d'appliquer hâtivement du ciment au sommet du mur, ce qui accroît le poids et accélère le processus, il convient de rétablir la cohésion des couches. Cet exemple est cité en connaissance de cause : sur beaucoup de chantiers, on se contente de cette solution de fortune qui est inadéquate et, de plus, inesthétique.

Si l'enduit ou le mortier se détachent l'un de l'autre ou du support, après avoir relevé les zones de poches d'air, on injecte à la seringue, d'abord, de l'eau et de l'alcool pour mouiller les parois de la poche, puis un mélange de caséate de chaux, avec $10 \%$ d'acétate de polyvinyle ${ }^{*}$, en profitant des crevasses éventuelles ou en forant des trous à des endroits choisis pour ne pas endommager la peinture. Le caséate sera d'abord assez liquide, puis plus épais, avec addition, si la poche est vaste, de poudre de marbre ou de sable. Pendant la durée de l'injection, la surface de la peinture est maintenue par des planches pour éviter que la pression exercée à l'intéricur ne la détache ${ }^{12}$.

\section{Dépose d'une peinture murale en place, mise sur un nouveau support}

Il faut choisir la méthode de transposition la mieux adaptée au cas d'espèce. Il y a deux principes, dont un présente une variante : les Italiens les nomment, strappo et stacco:

le strappo (de l'italien strappare, déchirer) consiste à coller de la gaze sur la peinture murale à déposer, puis à l'arracher rapidement, ce qui entraîne avec elle uniquement la pellicule picturale. Ce procédé n'est pas utilisé pour les peintures murales romaines mais pour les véritables fresques du Moyen Age et des Temps modernes; il est très pratique pour traiter les surfaces courbes, comme les voûtes. Le stacco (de l'italien slaccare, détacher) consiste à coller des toiles sur la peinture murale puis à détacher par derrière la peinture avec la couche d'enduit immédiatement sous-jacente et le stacco a massello, à détacher tout le support de mortier, qu'on fait basculer sur un panneau de bois prévu à cet effet.

De ce principe découlent toutes les méthodes de dépose des peintures romaines qui varient selon les pays. Il y a deux méthodes, l'une, traditionnelle, mise au point en Italie, l'autre utilisant des produits modernes et sensiblement différente en Italie et en Allemagne, les deux principaux pays à avoir fait des recherches systématiques dans ce domaine. Nous exposerons ces deux manières de procéder en soulignant les avantages et les inconvénients de chacune d'elles. Puis, nous aborderons l'expérience de restauration tentée sur une grande peinture dépassant $7 \mathrm{~m}$ de long, entreposée au musée de Soissons.

11 I. V. BoRRhila, op. cit., p. 339.

12 P. PHILPPot, P. Mora, op. cil., p. II, 2k. 


\section{A. Méthode traditionnelle italienne.}

1. Première phase: la dépose. - Après avoir effectué les analyses assurant qu'il s'agit bien d'une fresque, donc d'une peinture résistante à l'eau, puis l'avoir ensuite nettoyée, fixéc et consolidée, on est en mesure de commencer la pose des loiles. On aura divisé la peinture en autant de panneaux qu'il est nécessaire, car cette méthorke ne permet pas le détachement de trop grandes surfaces.

a) Pose des toiles. - Deux toiles sont collées sur les peintures à traiter. à l'aide d'une colle d'ébéniste, cervione en italien ; le cervione est constitué de colle forte d'os et de mélasse, ajoutée pour rendre la colle plus élastique, dans le rapport quatre pour un (par exemple $3 \mathrm{~kg}$ de colle pour $750 \mathrm{~g}$ de mélasse). Au cervione est joint du fiel de bouf $(250 \mathrm{~g})$, pour que la colle adhère mieux, du vinaigre de vin (2 litres) et du pentachlorophénate de sorlium pour neutraliser l'action des moisissures. Le tout est maintenu chaud au bain-marie, pour rester fluide : c'est la fameuse collela.

La première toile est une gaze, lavée pour lui enlever son apprêt, puis séchée; elle est découpée en bandes que l'on colle, en les faisant chevaucher, à la surface des peintures, avec la collela. Lorsque cette gaze est bien sèche, une seconde toile plus épaisse, toile de jute à trame assez large, est appliquée avec la même colle. On a soin de commencer par en bas pour qu'il n'y ait pas de coulée de colle sur la peinture.

b) Détachage de la paroi. Il faut entailler la paroi le long de la surface à détacher et la marteler avec un maillet en caoutchouc pour amorcer la séparation entre le mortier et le mur. Ensuite, une planche au format de la peinture à enlever est disposée sur le mur, après qu'on ait interposé du papier pour éviter que la colle de la seconde toile n'adhèro au bois; on y cloue les deux toiles qui dépassent largement au-dessus de la zone peinte ${ }^{13}$; puis, à l'aide de lames très longues, le restaurateur détache le mortier et la peinture recouverte de ses toiles du mur sur lequel ils s'accrochent. Il a soin de commencer par le bas, sur le côté, et non par en haut, car les gravats s'accumuleraient dans une poche et gêneraient le détachage ; il doit également s'efforcer de travailler toujours parallèlement au mur pour ne pas abîmer les peintures.

Les nécessités de ce travail d'adresse, exigeant l'enlèvement de petites portions de peintures, constituent un handicap lorsqu'il s'agit de traiter des murs entiers. L'habilete des restaurateurs italiens est telle qu'ils parviennent à préparer des plans de travail de plusieurs mètres à la fois.

c) Eliminalion du support de mortier. Après avoir fait basculer la peinture sur la planche destinée à la recevoir, il convient de gratter le mortier qui s'y trouve, pour ne laisser que $0, \bar{y} \mathrm{~cm}$ d'épaisseur en moyenne. Si le mortier est résistant, on peut lui laisser un centimètre, dans le cas contraire il faudra le diminuer davantage et ne conserver que la dernière pellicule superficielle teintée par la couleur. On épargne cette mince couche de mortier, généralement plus blanche et plus fine que les autres, afin de ne pas troubler l'environnement immédiat de la couleur et de peur de changer ou d'affaiblir le ton. Pour amincir le mortier, on peut 
employer des ciseaux à bois ou des burins et procéder par petits coups obliques. On enlèvera soigneusement la poussière de chaux et de sable, que l'on gardera pour la fabrication du nouveau support (fig. 15).

2. Seconde phase: le remplacement du support. - Lne fois la peinture grattée, les endroits qui ont perdu leur enduit peint apparaissent, ils sont comblés de sable pour éviter que le nouveau mortier, qui sera coulé, ne passe au-dessous et ne recouvre les surfaces peintes aroisinantes.

a) Fixalif. Au revers de la peinture dépouillée de la majeure partie de son mortier, on étend une couche de lait ou une émulsion acrylique moderne : le Primal AG 33 (marque Monte Catini).

b) Caséale de calcium. L'ne couche de caséate de chaux est appliquée; elle est obtenue en mélangeant un volume de caséine de lait étendue d'eau à trois volumes de chaux éteinte*.

c) Châssis. Il est disposé sur cette couche de caséate de chaux pour servir d'armature au nouveau support. Les compartiments métalliques formés par les barres transversales ne doivent pas excéder $50 \mathrm{~cm}$ de còté. Le tout est protégé contre la rouille.

d) Mortier. Le grillage est recouvert d'un mortier constitué d'un volume de caséate de chaux pour deux volumes de poudre de marbre, trois de sable de rivière lavé et trois volumes de chaux éteinte. l'ne seconde couche de ce nouveau mortier est étendue pour éviter la formation de fissures. On peut utiliser l'ancien mortier pour épaissir la préparation. Pour empêcher la formation de craquelures, le mortier doit être retravaillé, lorsqu'il commence à prendre. On mouille à nouveau la surface et on la recouvre de sable sec et propre.

3. Remise à nu. - Au bout d'un mois de repos, la peinture ainsi traitée est retournée, la planche est enlevée, les deux toiles sont retirées avec de l'eau chaude. La gomme-laque, lorsqu'elle a été employée comme fixatif, est remplacée par le Paraloïd. La restauration est complétée, si hesoin est, par un tracé discret, un simple trait gravé, parfois coloré pour que l'œil puisse suivre les lignes du dessin, surtout lorsqu'il s'agit d'architectures peintes. On peut aussi maquiller les vides laissés par les peintures disparues à l'aide de couleurs de tons proches, nettement distinctes des peintures originales : ce sont de simples traits parallèles de coulcurs, qui éliminent l'impression désagréable de trous blancs au milieu d'une composition. En aucun cas, il ne faut se permettre de repeindre certains motifs ou des champs unis qui se sont estompés à la restauration. Le maquillage doit être discret et distinct des fragments subsistants. Les peintures, replacées dans la pièce dont elles proviennent, chaque fois que c'est possible, sont isolées du mur par un espace de dix centimètres pour les défendre contre la stagnation de l'humidité et permettre une bonne ventilation au revers du nouveau support.

Les qualités et les défauts de cette méthode sont bien connus : elle n'endommage absolument pas les peintures, même si elles sont en piteux état, et des résultats durables ont été ainsi obtenus. Aussi son avantage majeur est-il d'avoir été largement expérimenté, et d'offrir toute sécurité en ce qui concerne la bonne conservation des peintures traitées.

Cependant les nombreux inconvénients qu'entraîne l'emploi de produits naturels, visant à reconstituer un support identique à l'original, ont conduit les services de restauration à chercher d'autres méthodes. En effet, les opérations sont très longues, la préparation 
de la colle exige beaucoup de soin (il faut la maintenir chaude) et de savoir-faire, le temps de séchage entre les différentes phases est assez considérable (un mois après l'application du nouveau support), les manipulations elles-mêmes sont lentes, comme le collage des bandes de gaze une par une. Enfin, elle nécessite le recours à des maçons professionnels pour appliquer le nouveau mortier, à cause des risques de crevasses. Surtout, on doit souligner le poids énorme des panneaux ainsi refaits, qui sont très lourds et intransportables, même pour de petites surfaces, ce qui oblige à fragmenter beaucoup trop un mur peint entier à déposer. Signalons qu'un succédané de cette méthode a été essayé en France. On a souvent utilisé, et on l'utilise encore malheureusement, du plâtre pour fabriquer le nouveau mortier. Le plâtre fait des ravages en voilant les couleurs jusqu'à les faire disparaître complètement ${ }^{14}$. De plus, il n'adhère pas au mortier et les reconstitutions faites ainsi ne tiennent pas. Certains ont cru bon également d'utiliser du ciment : son emploi est déconseillé à cause des efflorescences salines et des nitrates qu'il provoque, conduisant à un ternissement de la couleur. qui s'assombrit graduellement ${ }^{15}$.

\section{B. MÉthodes modernes.}

L'emploi de produits synthétiques a permis d'alléger considérablement les supports des peintures détachées; c'est la différence essentielle avec la méthode traditionnelle ${ }^{16}$.

1. Méthode ilalienne. -- La première phase du travail est identique, la dépose de la peinture se fait comme par le passé mais la seconde phase, le remplacement du support. est totalement différente, faisant appel à des matériaux plastiques expansés. Cielui qui semble avoir donné le plus de satisfaction est le polystyrol expansé*, qui sert d'élément central dans les structures en sandwich des supports des peintures détachées. Voici les différentes opérations nécessaires pour constituer ce support:

a) Fixalif. A l'arrière de la peinture réduite à une mince épaisseur, on fixe la couleur, comme dans le procédé traditionnel, par l'émulsion acrylique déjà citée : le Primal AC 33.

b) Applicalion des loiles. Un voile de coton est collé au-dessus, au caséate de chaux, puis une toile, de la même façon.

c) Panneau de plastique expansé. sur cette toile est ajustée au caséate de chaux une feuille de polystyrol expansé commercial (frigolit), taillée à la mesure voulue, d'une épaisseur de $5 \mathrm{~mm}$; on étend par-dessus une seconde toile.

d) Vernissage. Le vernissage est opéré à la gomme-laque en solution alcoolique.

14 L.es perintures de Nizy-le-Comte, dont certains dessins nous ont éte conservés, nous font regretter les traitements nocifs qui leur furent infligés. Certains fragments furent "séchés de suile, tranquillement un à un, wt devant un feu doux helas', couverts à temps d'un inoffensif vernis à l'ouf " mais pour les grands panneaux, l'auteur reconnaît. de lui-même : - les grands panneaux lont été], bardés de plâtre absolument à déconseiller! et conservés pendant dix ans dans ce milieu destructeur avant d'être insérés dans les murailles de ce musée. Pendant cinq ans il a constamment poussé à la surface peinte des efflorescences nitreuses qu'on n'a jamais pu arrêter. Très clairs et lisibles apris les lavages, ils noircirent de plus en plus. Le reslaurateur de la mosaïque de Nizy crut bien faire en passant les peintures au vernis d'essence grasse. Lopération ne fit que les assombrir davantage. Aujourd hui, il est diflicile de les lire ". L. Fl.Et kr, Antiquilés el monuments du département de l'Aisne, Paris, 1878, II, p. 11-12. Sous ajouterons quacluellement on ne distingue plus rien.

15 L. V. Воннал..., op. cil., p. 339.

16 P. Mora, G. Torraca, annexe au Rapporl sur les peintures murales, ICOM, 1965, p. 1 et s. 
e) Consolidalion. On applique une couche de polyester et de fibre de verre (mat) de $300 \mathrm{gr} / \mathrm{m}^{2}$, puis on construit une ossature en profilé d'aluminium anodisé, fixée sur la couche de polyester renforcée avant polymérisation. Le tout est recouvert de bandes de fibres de verre imbibées de polyester. Toutes sortes de précautions, qui multiplient les opérations, sont rendues nécessaires par la nature du polystyrol. Par exemple, le vernissage à la gomme-laque a pour objet d'imperméabiliser le polystyrol expansé et d'éviter que le styrol contenu dans le polyester appliqué au-dessus ne le dissolve. Le polyester lui-mème (5126, résine à temps d'emploi prolongé) a l'avantage d'ètre ininllammable et évite le grauchissement de la couche après durcissement.

L'intérêt remarquable de la méthode réside dans la légèreté du panneau ainsi fabriqué, car l'ensemble du support a une épaisseur de $2,5 \mathrm{~cm}$, si on ne tient pas compte de l'ossature métallique, qui doit avoir une épaisseur de $1 \mathrm{~cm}$ au maximum. Le poids d'un panneau d'1 $\mathrm{m}^{2}$ est de $11 \mathrm{~kg}$ à $12 \mathrm{~kg}$. Les inconvénients sont cependant très réels et tiennent à la fois à la nature du produit utilisé et aux qualités requises pour savoir l'employer. Comme le polystyrol expansé ne peut être exposé directement aux agents atmosphériques, particulièrement au soleil dont les rayons ultra-riolets provoquent le jaunissement et la dégénérescence, il doit être en sandwich dans un panneau. De plus, il est inflammable. I'où toutes les mesures de protection qui compliquent son emploi. Si nous laissons de côté le problème du vieillissement de ce type de support, que nous connaissons mal (bien que des expériences de vieillissement artificiel aient été faites en laboratoire), l'obstacle majeur à son emploi est le façonnement d'un panneau aux dimensions voulues, qui exige un savoir-faire de spécialiste et un encombrement important pour stocker les feuilles de plastique expansé. Enfin, le coût de l'opération est élevé, car beaucoup de produits différents entrent dans ce type de restauration.

Les laboratoires italiens cherchent d'autres supports et procèdent a toutes sortes d'expériences. Ils ont déjà éliminé certains matériaux plastiques ne présentant pas toutes les garanties voulues, toutes les qualités requises : ainsi le polyuréthane; en revanche, le chlorure de polyvinyle* expansé, moins sensible aux solvants que le polystyrol, est très résistant aux agents atmosphériques, aux micro-organismes, insensible à l'humidité. On réalise des supports, comme avec le polystyrol, ou à peu de chose près.

a) Fixatif. Ine couche de $3 \mathrm{~mm}$ est étendue au revers de la peinture débarrassée de son mortier superflu, composée de chaux, avec silice naturelle à poids spécifique bas (Perlite), renforcée par une émulsion vinylique (Vitravil).

b) Toile. Collée au caséate de chaux avec addition de Vitravil.

c) Panneau de plastique expansé. Un panneau de 10 à $12 \mathrm{~mm}$ d'épaisseur de chlorure de polyvinyle expansé (Cadorite) est ajusté, puis une dernière toile est rajoutée comme précédemment, collée à l'émulsion vinylique. $\Lambda$ part le cadre métallique, il n'y a pas d'autres opérations de protection.

Après ces méthodes difficiles à mettre en œuvre, car elles nécessitent un matériel d'équipement, des crédits et des spécialistes bien formés, nous allons examiner maintenant la méthode mise au point en Allemagne, notamment au musée de Trèves. qui nous a semblé de loin la plus praticable, bien que pour l'appliquer, une grande habileté manuelle, un soin extrême et minuticux soient tout aussi indispensables. 
2. Méthode allemande. - Cette méthode a l'avantage de convenir à l'état rles peintures telles qu'on les trouve au nord des Alpes, c'est-à-dire le plus souvent fortement humidifiées par la terre environnante et menacées d'une destruction très rapide en raison des agents atmosphériques. De ce fait, les solutions envisagées correspondent mieux aux besoins que nous éprouvons en France, où le climat est sensiblement le même. La phase de dépose diffère de la méthode italienne en ce que l'assèchement du mur portant la peinture est le premier souci qui intervienne ${ }^{17}$.

\section{Première phase: la dépose.}

a) Assèchement du support. Il est indispensable, pour que la colle adhere bien ì la peinlure. Pour ce faire, la solution adoptée consiste à disposer des lampes à infra-rouges le long de la paroi à traiter (chaque ampoule consomme $3 \mathrm{kw} / \mathrm{h}$ ) ; elles sont maintenues jusqu'à ce que le mur soit sec, en général pendant une journée. Les opérations suivantes sont analogues à la méthode italienne mais les produits employés raccourcissent les temps entre les opérations.

b) Pose des loiles. La colle appliquée sur le mur est synthétique et ne demande pas de longue préparation : il s'agit de Nowilith* 35/73, à 60\% en solution dans l'acétate d'éthyle qui peut ètre dilué dans de l'acétate d'éthylglycol si elle est trop visqueuse. I'ne gaze, synthétique aussi, préparée à l'Acronal, est étendue sur la paroi, sa tenue permet de l'utiliser telle quelle et non pas de la découper en bandes, comme pour la gaze de coton trop élastique. Au-dessus. une toile de colon à trame serrée est ajustée, pour renforcer et éviter que la colle ne bave sur la planche destinée à recevoir la peinture lors du détachage (ce qui rend inutile l'interposition de papier).

c) Détachage de la paroi. Les toiles sont clouées sur la planche et, après deux heures d'attente pour le séchage, on détache peinture et mortier du mur comme dans la méthode italienne.

d) Élimination du mortier. Elle se fait comme en Italie, a l'aide de ràpes ou de ciseaux à bois, pour ne conserver que la mince couche blanche supportant l'enduit peint.

\section{Seconde phase: remplacemenl du support.}

a) Fixalif. Il a pour but de durcir la couche de mortier restant. On ulilise l'llermétique Conservans* ; si besoin est, il peut être dilué dans l'eau. Il faut laisser ensuite sécher un à deux jours.

b) Applicalion d'un mortier sunnthétique. Ce mortier, facile à fabriquer, est constitué de :

trois parts de .lowilith D),

une part de .lowilith D 025.

quatre parts d'eau,

sable de rivière (en général au moins 16 parts) et un peu de poudre calcaire. en quantité suflisante pour obtenir une pâte malléable qui ne coule pas.

Ce mortier est posé en couche très mince $(0,5 \mathrm{~cm}$ à $1 \mathrm{~cm})$. (On y applique une gaze synthétique pour rendre le tout bien homogène, l'ensemble est généralement sec au bout de trois à quatre jours, une semaine au maximum.

c) Opéralions finales. L'ensemble une fois retourné, on peut enlever les deux tissus prolégeant la peinture à l'aide d'acétate d'éthylgrlycol. Le peu de Nowilith qui subsiste sert de fixatif à la peinture. S'il en reste un peu trop et qu'une brillance soit visible, il suffit de frot ter les endroits trop luisants avec l'acétate d'éthylglycol. l'ne armature métallique est alors ajustée sur le pourtour du panneau. On peut exécuter ensuite des retouches pour combler les vides de la peinture.

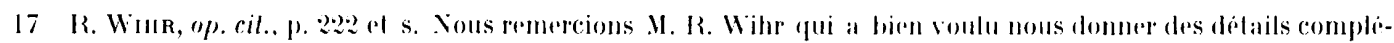
mentaires of nous Jui sommes reconnaissante de nous permetle d'utiliser larerment les remarques qui lui ont ele dictées par sa large expérience. 
Les avantages du procédé utilisé en Allemagne sont très nombreux. L'emploi d'une colle synthétique simplifie beaucoup les opérations préliminaires. de même l'utilisation d'une gaze synthétique rigide, très solide, qui se déforme peu. La facilité de confection du mortier nouveau est à noter, l'emploi de sable de rivière lui donne unc texturc analogue au mortier originel, cr qui permet de le laisser tel quel, lorsque des retouches pour de petites lac'unes ne s'imposent pas. De plus, il n'a pas besoin d'être appliqué sur une grande épaisseur. Un panneau(de $2 \mathrm{~m} \times 2,50 \mathrm{~m}$ ), peu épais, ne dépasse pas $75 \mathrm{~kg}^{13}$, armature comprise, ce qui rend un transport tout à fait praticable. Remarquons aussi que le laps de temps pour le séchage est très court : une semaine au maximum, au lieu d'un mois dans le procédé traditionnel. Comme toujours, le seul inconvénient est le manque de recul pour juger de l'inaltération des qualités de ces produits synthétiques mais, là encore, des tests de laboratoire ont été satisfaisants : les réalisations du musée de Trèves, dont certaines ont déjà dix ans d'existence, sont une première appréciation très favorable pour cette méthode relativement aisée à appliquer. Ajoutons que ces produits ne sont pas d'un coût exagéré.

Néanmoins, le fait que le solvant de la colle Mowilith soit aussi celui du mortier synthétique (à base de Mowilith également) est gênant car, au moment d'enlever les toiles, le mortier se ramollit et peut même se détériorer, ce qui exige une réfection. Nous verrons que cette colle présente des inconvénients lorsqu'il s'agit de recomposer des fragments.

\section{Recomposition et pose sur un support de fragments peints}

La technique utilisée est dérivée de la méthode allemande, son adaptation au cas très particulier des fragments de peinture de Mercin-et-Vaux (Aisne), conservés au musée de Soissons, a posé de très sérieuses difficultés, qui sont loin d'être toutes résolues. Outre le fait que nous avions à notre disposition des crédits limités et une équipe d'étudiants bénévoles ${ }^{19}$ non préparés, nous nous trouvions en présence de fragments récupérés d'une fouille non autorisée. complètés par tous les débris trouvés par nous-mêmes devant le mur de la pièce qu'ils décoraient (fig j̃). Cies circonstances, l'état fragmentaire de la peinture, sont le lot commun en France; c'est pourquoi nous croyons que cette expérience sera profitable à d'autres.

a) Mise sur sable de la reslitution. La première difficulté était de trouver le moyen de reconstituer le décor se développant sur 7,28 $\mathrm{m}$ de long et 3,40 $\mathrm{m}$ de haut, en autant de panneaux nécessaires à la mise sur un nouveau support. Les panneaux les plus grands ne dépassent pas $4,80 \mathrm{~m}^{2}$, ce qui est déjà important. Le second problème consistait à trouver un support assez élastique et résistant à la fois pour poser tous les fragments, de manière à obtenir une surface peinte bien plane, permettant ultérieurement à la colle et à la gaze

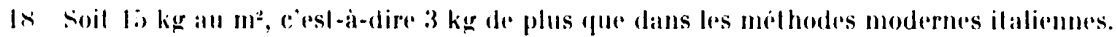

19 Nous temons a remercier tous ceux qui ont participé aux stages à Soissons et plus spécialement b. (:harnier, (.. Daniau, L. Dartiguenave, II. Fristov, If. Fourmont, M. Fabioux, F. Godard, M. F. Gleizes, D. Jaillot, S. Martigne, C. Nerzic, D. Picard, D. Pette, F. Saint-Jean, . I. C. Tansorier, P. Voute, C. Wilmotte el le Club d'archeologie du 67e regiment d'infanterie de Soissons. Notre reconnaissance va egalement a $\mathrm{M}$. E. Will, instigateur de celle entreprise. 


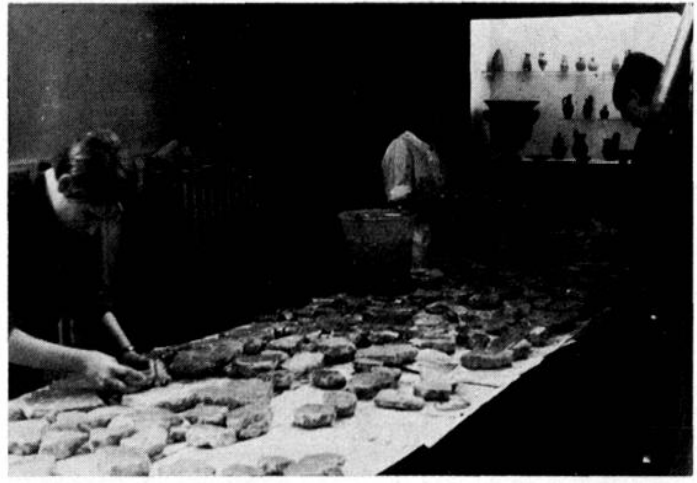

5) Nettovage et recomposition des peintures de .Mercinet-Vaux, au musée de Soissons.

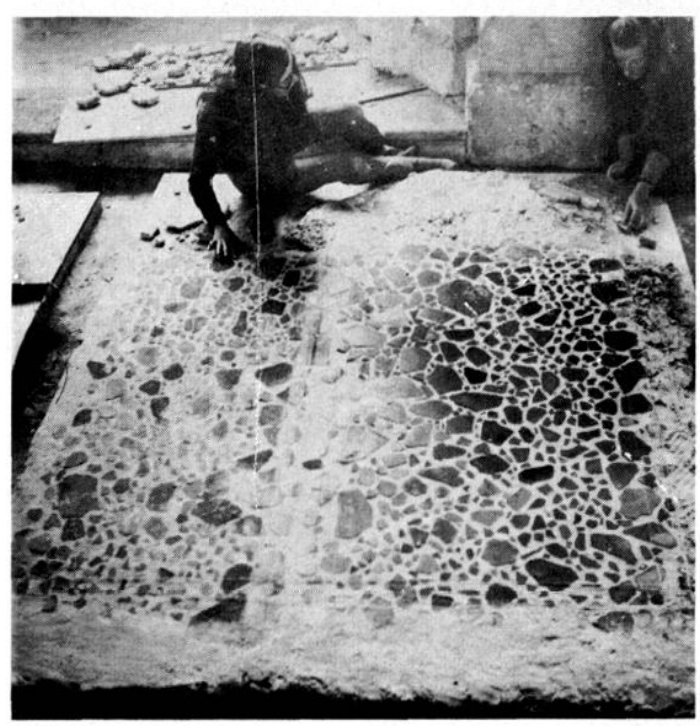

7 Restitution du décor d'un orthostale rouge à colonnes et fronton de la peinture de Vercin-et-laux panneall de $1,9 \times \mathrm{m} \times 2,20 \mathrm{~m}$. Des ficelle's sonil lendures pour bien aligner les decors.

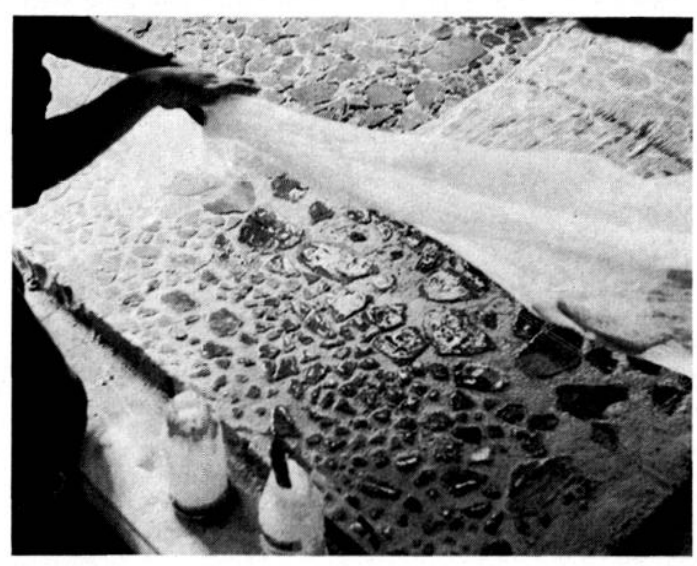

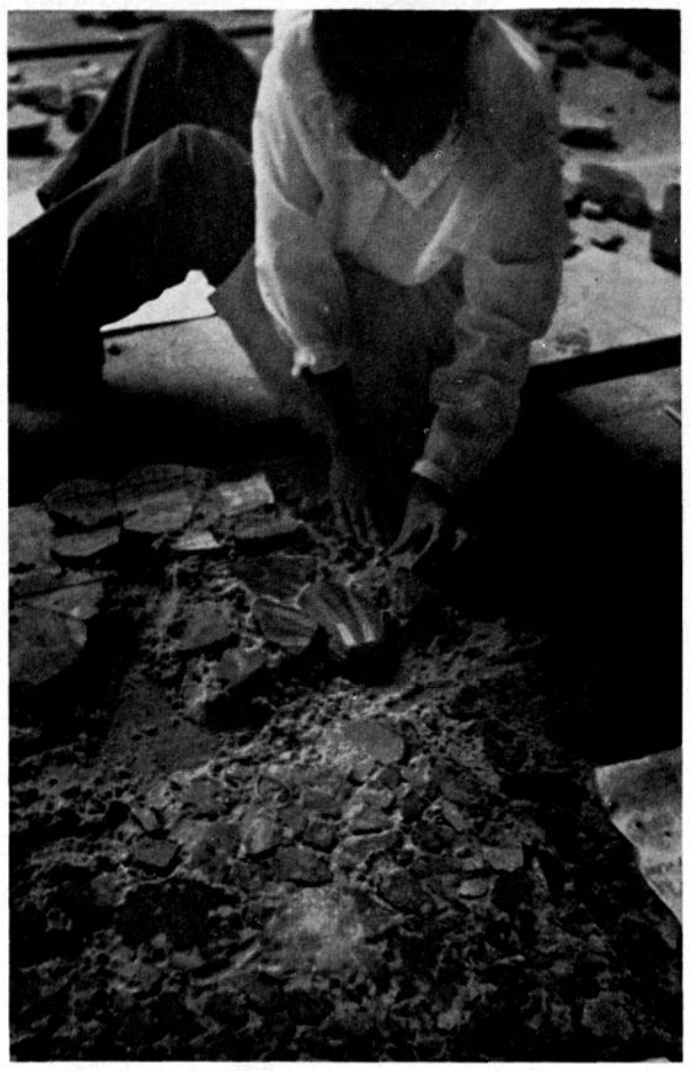

6) Reconstitution du decor de lat plinthe de la peinture de Hercin-ed-Vaux. La salle, qui cale les morecaux d'epaissenrs differentes, est ajoute an fur el à mesure.

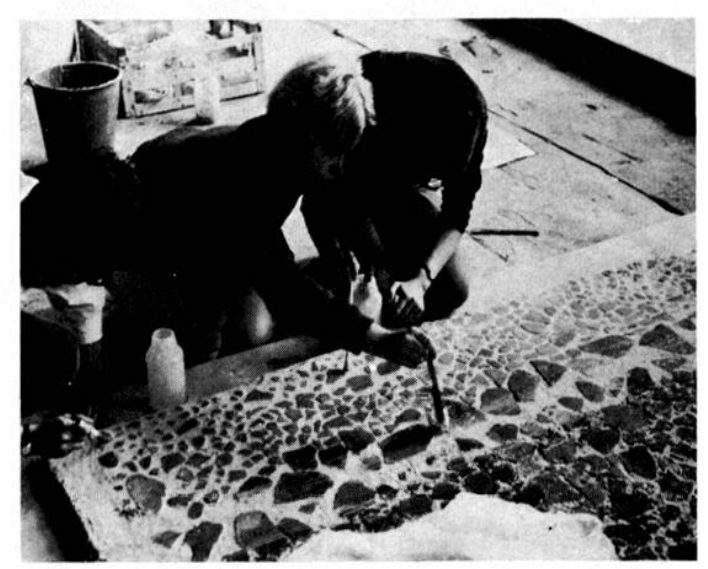

$\times$ Preinture de Mercin-et-Vaux, musée de Soissons; encollage de la pellicule picturale.

<- 9 Peinlure de Mercin-el-Vaux, anloilage des morceaux de peinture encollis. 
de bien adhérer sur tous les morceaux. Nous avons choisi de recouvrir les planches de sable fin, humidifié pour qu'il ait assez de tenue et pour que nous puissions enfoncer commodément les fragments, selon leur épaisseur respective, afin d'avoir une planéité parfaite (fig. 6 et 7 ).

b) Pose des loiles. La colle (Mowilith 35/73) fut appliquée soigneusement sur tous les morceaux de peinture et, en l'occurrence, nous avons remarqué que les débris infimes étaient inutilisables car ils collent au pinceau et s'en vont (fig. 8). Puis, la gaze synthétique, plus épaisse que celle qu'on utilise en Allemagne, a été étendue, en prenant garde à ne pas faire de plis disgracieux, ce qui est très difficile (fig. 9). Par précaution, après un petit temps de séchage, une nouvelle couche de colle a été passée au-dessus de cette gaze (fig. 10). Au-dessus, on a tendu la toile de coton à trame serrée, en la faisant largement dépasser sur les bords et en la fixant avec des punaises à la planche (des clous enfoncés au marteau auraient risqué, en ébranlant les morceaux, de les abîmer et de les déplacer) (fig. 11). Après avoir observé, sur les premiers panneaux traités, que la toile, malgré nos soins, se détendait et faisait des plis en laissant une empreinte en creux au moment du coulage du mortier, nous avons essayé de la mouiller, pour qu'en séchant clle se tende mieux.

c) Retournement. Afin de retourner sans crainte l'ensemble du panneau, nous avons appliqué une seconde planche sur les deux toiles, en serrant la peinture entre ces deux planches au moyen de presses. Après un temps de séchage de huit jours au minimum, nous avons fait procéder au retournement des panneaux (fig. 12). Malgré le pouvoir adhésif très fort de la colle, certains morceaux s'étaient détachés. C'est pourquoi il faut toujours faire un calque des contours des morceaux et de leurs couleurs, pour être en mesure de replacer à l'envers les fragments qui ont glissé. Leur proportion reste d'ailleurs très faible. Nous avons essayé d'attendre plus longtemps que la colle prenne, avant de retourner les panneaux mais celle-ci durcit trop et la pellicule picturale des fragments en souffre au moment de la mise à nu. De plus, la toile pourrit si on attend un mois.

d) Préparalion de la peinlure à son nouveau support. Lorsque le panneau est retourné et que la planche inférieure, maintenant située au-dessus, est enlevée, il faut retirer tout le sable qui a servi de support aux fragments lors de la restitution (fig. 13). Une fois les fragments bien nettoyés, on coule une mince couche de mortier synthétique autour de chacun d'eux, ne dépassant pas $2 \mathrm{~mm}$, pour empêcher que les bords ne s'effritent sous les coups de burin au moment de l'élimination du mortier superflu (fig. 14). Il faut bien faire attention à ne pas en laisser tomber sur des fragments car ce mortier de Mowilith, dont la formule a été donnée plus haut, devient très dur et est très difficile à enlever. $\mathrm{Au}$ bout d'une journée ou deux, on procède au burinage du mortier romain en enlevant la poussière avec des brosses et un aspirateur (fig. 15). Puis, on badigeonne les fragments d'Hermétique Conservans pour les durcir.

c) Applicalion du morlier synthétique. (ce mortier, fabriqué comme il a été conseille ('i-dessus, a èté appliqué en trois couches au moins pour obtenir une épaisseur totale variant de 1,8 à $2,2 \mathrm{~cm}$ selon les panneaux (fig. 16). Il faut éviter absolument qu'une partie liquide ne surnage, car elle durcit en une mince pellicule qui n'arhère plus à l'ensemble ; il faut 


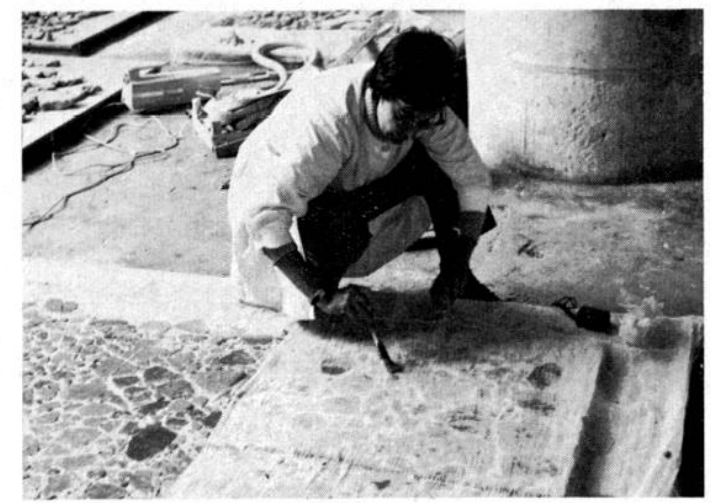

10) Peinture de Merein-el-Vanx. Ine conche de colle est rajoutio all-dessus de la garre.

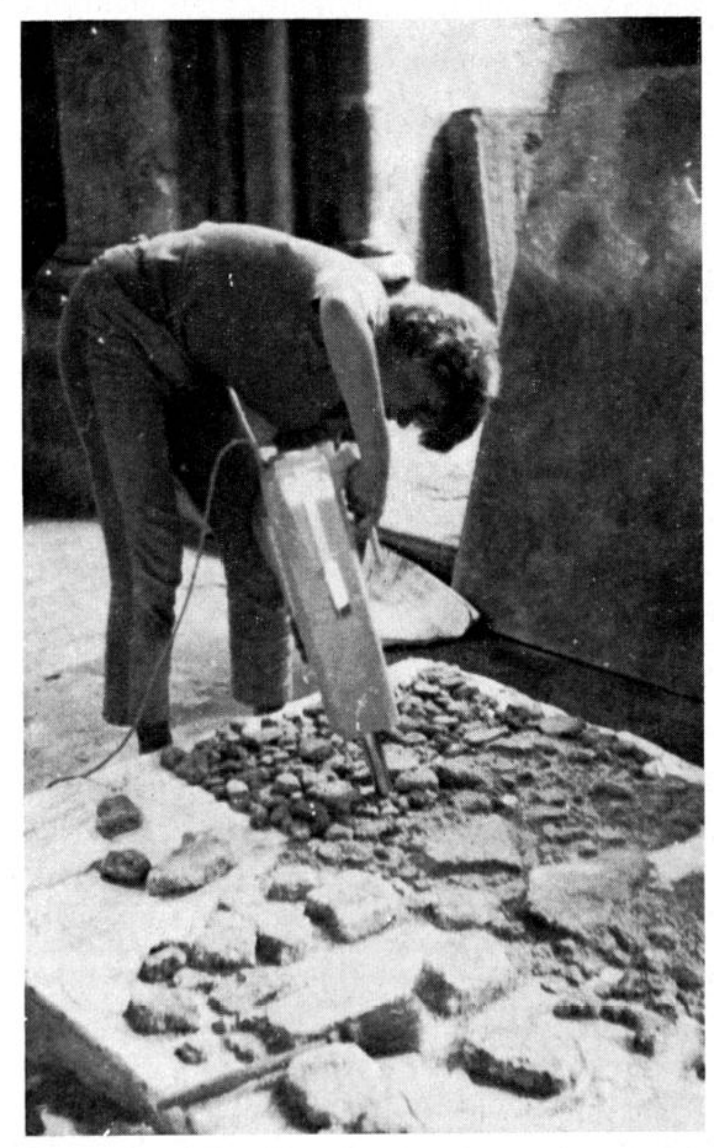

13 Peinture de Mercin-el-lianx. Aree un aspirateur

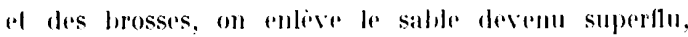
qui a servi de support anx fragments de peinture.

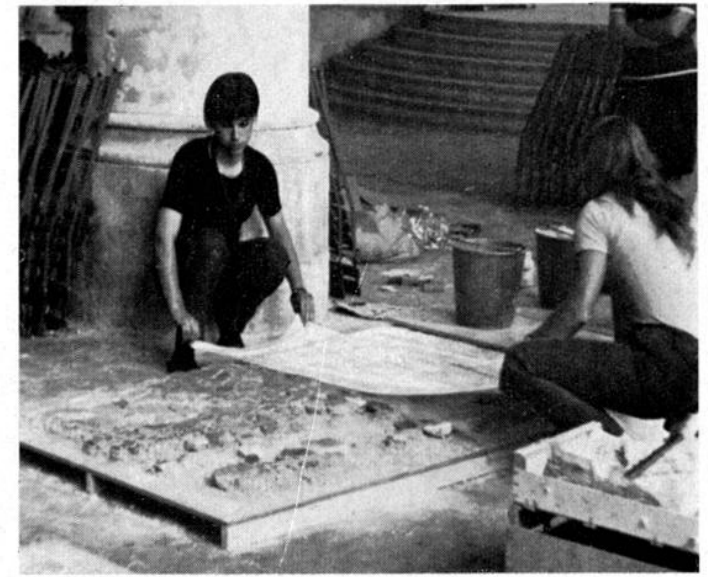

11 Pose de la seconde loile a trame serrée sur la peinture de Mrercin-et-Vaux.

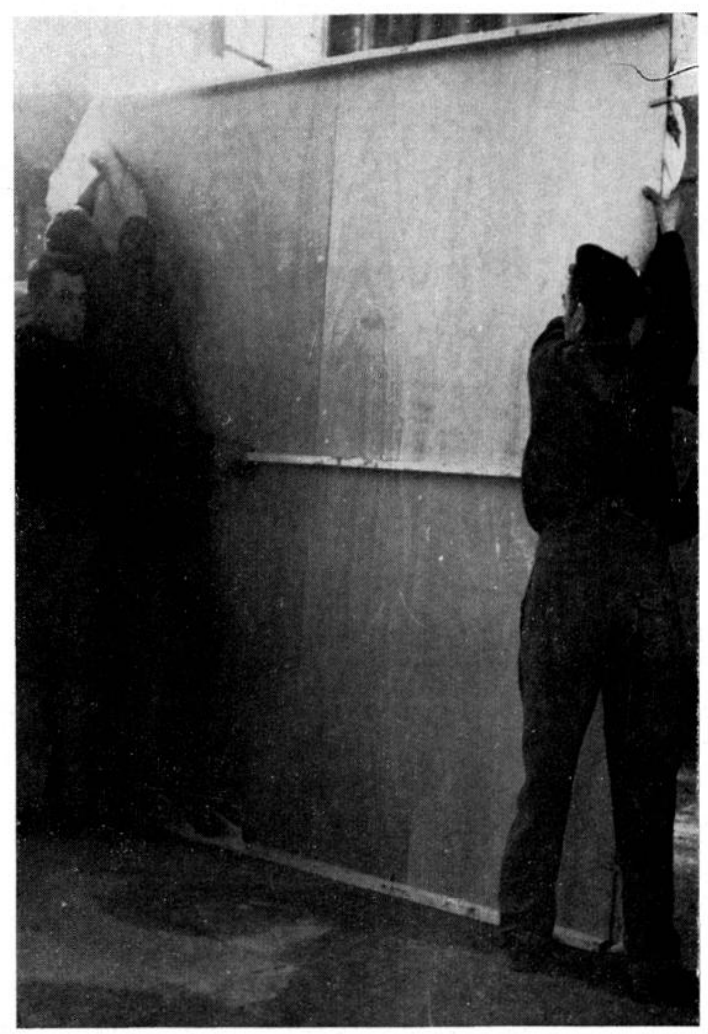

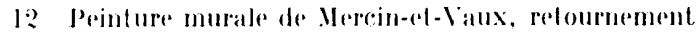
d'un des grands pamneatux. (On apereoil sur les cotes les presses qui serrent les deux planches intere lespuclles se trouvent les fragments de peinture. 


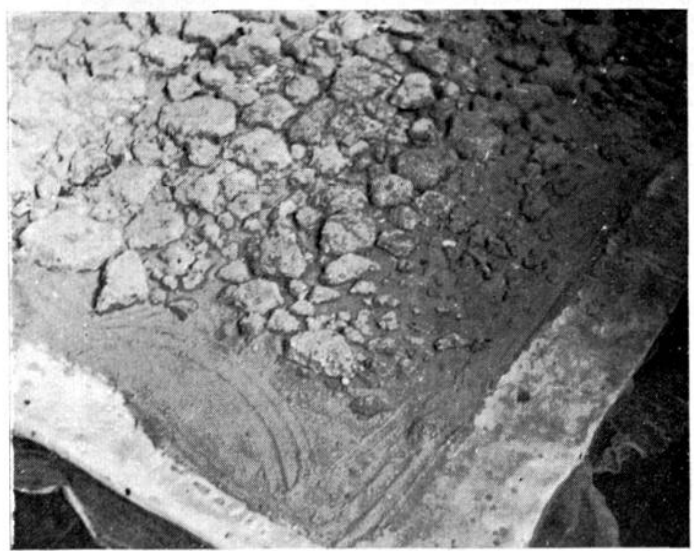

14 Printure murale de Merein-el-1:aus. fone mince couche de mortier stntheligure est coulere pour protener les bords des fragments de peinlure. lin bas a droile,

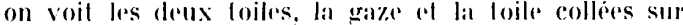
la perinture, dont la face se trouse contre terre.

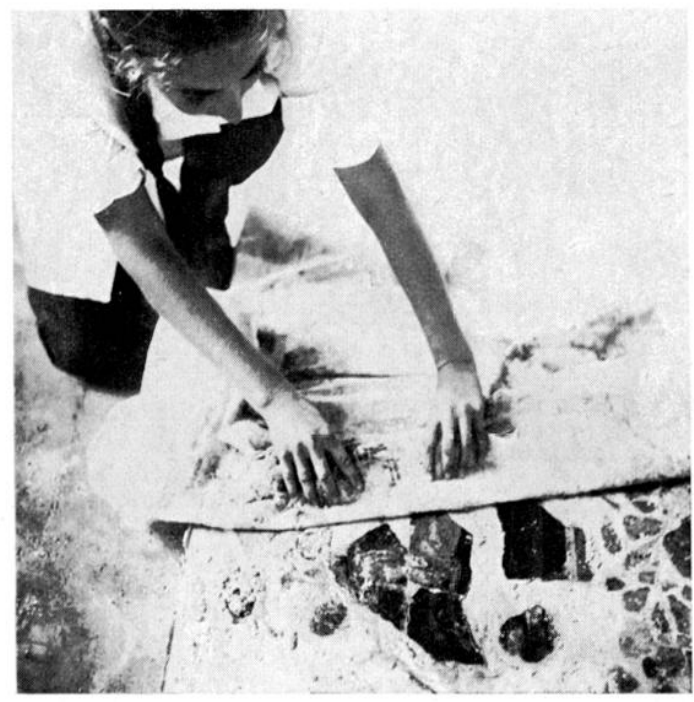

17 Peinture murale de Mercin-et-Vaux. Apres retournement du panneatu, la gate al la toile sont entevies aree un solvant, une secone de chasse apparait, dont on voit iei un petil cheval; plinthe.

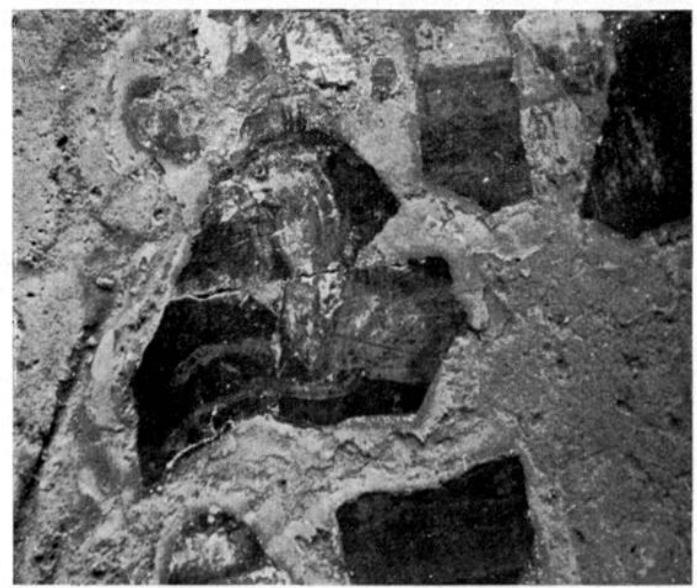

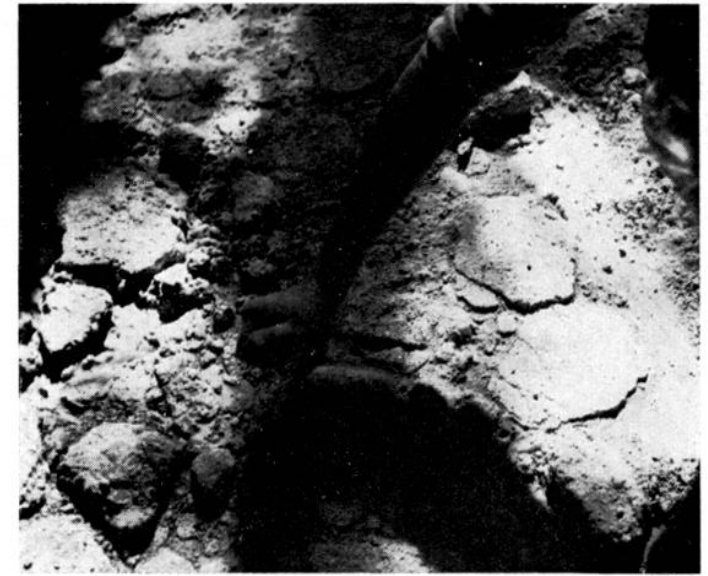

15) Peinlure mumate de Mercin-en-lants. Burinage des morticrs au revers des fragments de printure. On apereoit: a droite en haul, des frangements dija désepaissis, reduils a quelques millimetres ; a ganche en bres, on distingue difrerentes couches de mortier qui vont etre enleveres à leur tour.

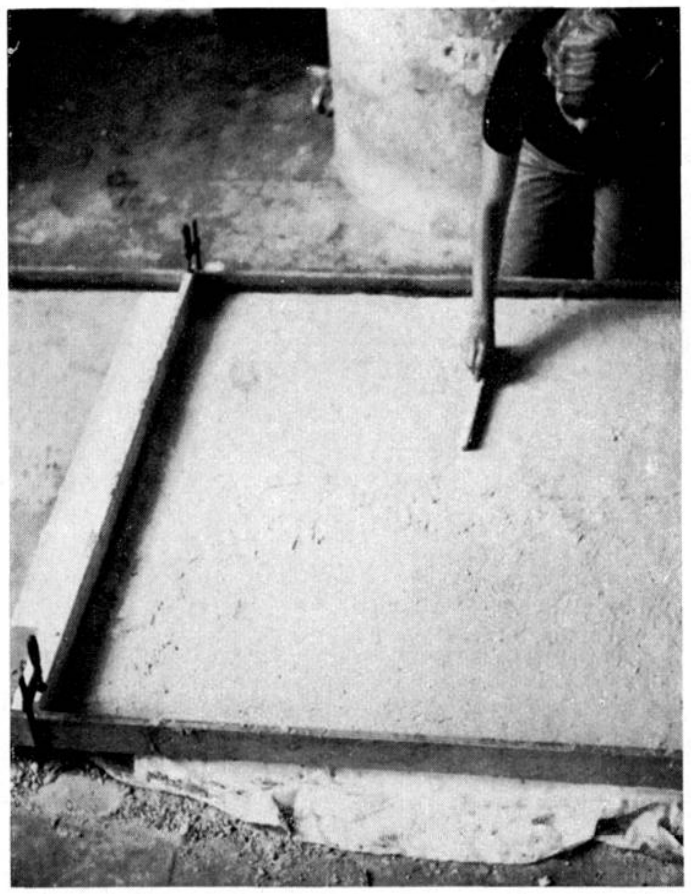

16 Peinture murale de Mercin-ert-lanux. Plusieurs conches de mortier ssunthetigue sont conlíes of lissées, an revers des peinlures éminciens.

\&- Ix Peinture de Mercin-el-Vans. Detail du pelit cheval de la frise de chasse. II me reste plus qu à regaliser la surface oir des empreintes de plis de la toile sont

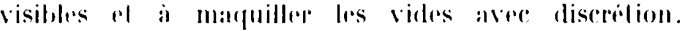


donc, au besoin, parsemer modérément la dernière couche de sable sec et appliquer une gaze synthétique finale, pour solidariser la totalite du panneau.

f) Mise à nu de la peinlure. Nous avons eu des difficultés particulières à décoller la toile et la gaze, car l'acétate d'éthylglycol, solvant employé, agit très lentement et la force d'attraction de la colle est telle qu'elle arrive parfois à arracher la pellicule picturale de rertains morceaux trop petits, ou, semble-t-il, exécutés dans une fresque mixte avec liant, re qui les rend très fragiles. Certains panneaux avaient été encollés de gaze de coton, au lieu de gaze synthétique qui nous faisait défaut. A cette occasion, nous avons pu constater qu'il etait imprudent de le faire car la gaze naturelle s'enfonce et se dissout quasiment dans le mortier synthétique qui remplit les lacunes entre les fragments de peinture; il est alors impossible de l'enlever à ces endroits-là et difficile de le faire sur les morceaux peints. Il n'est pas douteux qu'il faut adapter cette méthode au cas particulier, très répandu, des restitutions de peinture à partir de morceaux souvent exigus, qui ne se recollent pas entre eux. L'emploi d'un autre solvant de la colle, l'acélone, n'est pas idéal. Celui-ci ne décolle que les toiles et laisse à la surface de la pellicule picturale toute la colle durcie.

Les couleurs les plus délicates à conserver sur la peinture de Mercin sont le vert clair ct le jaune. Néanmoins les résultats de ce type de traitement sont intéressants (fig. 17) pour la majorité des couleurs el des morceaux. Autre désagrément, qui disparaîtra avec une plus grande habitude de la restauration : les plis de la toile qui s'impriment en creux dans le mortier synthétique et qui obligeront à un maquillage minutieux pour aplanir la surface (fig. 18). De tels défauts n'apparaissent évidemment pas lorsqu'on traite des plaques ou des murs ayant très peu de lacunes. Même le problème de l'adhérence de la colle ne joue plus sur de grandes surfaces. L'intérêt de ce procédé est sa réversibilité : il est assez facile, en cas d'erreur, de dissoudre le mortier synthélique avee son solvant (acétate de Méthylglycol) et d'enlever les morceaux incriminés, puis de reboucher le trou. A l'inverse il nous est arrivé de trouver des morceaux importants (deux oiseaux) qui s'assemblaient avec un fragment déjà mis sur le support. Nous avons évidé l'endroit où se placent ces éléments, a l'aide du solvant, pour pouvoir les inclure.

Il est regrettable que nous manquions en France d'un centre de restauration de peintures antiques qui ferait toutes expériences nécessaires sur de nouveaux produits, à l'image de ce qui se pratique à l'étranger et que seules les peintures en place et spectaculaires puissent bénéficier des soins de restaurateurs privés. Or, l'histoire de la peinture murale en Gaule, telle que nous voudrions la retrouver, nécessite une conservation adéquate et urgente de tous les documents peints, qui ne se limilent pas aux seules scènes animées, aux seuls sujets figurés. Nous voudrions attirer l'attention sur cet aspect des vastes problèmes que pose cette branche de l'art antique, où la restauration et la recherche scientifique des techniques et des styles doivent marcher de pair. On trouvera, plus loin, en appendice, un glossaire des termes techniques qu'il nous a paru utile d'établir, en raison de la grande confusion qui règne encore à cet égard.

L'expérience menée, en opérant le sauvetage de peintures, réduites à des débris, d'un établissement romain près de Soissons prouve que la restauration est indispensable aux 
disciplines archéologiques ${ }^{20}$. C'est elle qui nous a permis de restituer un décor qrandiose de frontons encadrés de colonnettes, toute une série de scènes animées de chasse, des paysages nilotiques, se développant tout autour d'une salle n'ayant pas moins de $7,50 \mathrm{~m}$ sur $12 \mathrm{~m}$. Elle seule est en mesure de nous fournir des critères de datation et d'apporter des éléments solides pour la connaissance de la peinture murale romaine en (iaule ${ }^{21}$.

\section{Alix BARBET.}

\section{Glossaire des trRmes technigues}

Crépr. (ital. intonaco ou incumicialura). Sorte de mortier* fabriqué avec du sable grenu et de la chaux* (ou du plàtre. du mortier de ciment), fouetté contre les parois d'un ouvrage, soit à la truelle, soil au balai ${ }^{22}$, sur le parement extérieur. Il est incolore ou coloré mais n'est. jamais peint. Le pas confondre avec l'enduit* peint ou le stuc* (voir ces mots).

DÉtrimpe (ital. guazzo). La détrempe est une variété de tempera* (voir ce mot), une préparation aqueuse, plus ou moins riche en adhésif ; c'est un liant* organique iexcepté la colle animale). lille est tries utilisée pour les plafonds et les murs à l'abri de l'humidité.

ENDUt Phent (ital. lonachino). L'enduit* est la pellicule de surface d'un mortier*. en général lissée ; c'est un mélange de chaux* et de calcite (ou de poudre de marbre), sans sable en principe. sur lequel est appliquée la couleur. Ye pas confondre avec le stuc* qui n'a pas la même composition et qui est en relief (voir ce mot). L'enduit blanc est dù :

a) soit à la couleur mème de la chaux*, qui est plus ou moins blanche;

b) soit à une couleur blanche rajoutée sur l'enduit même (lait de chaux* ou autre substance). Ce procédé est utilisé parfois lorsqu'on désire peindre quelques motifs légers sur l'enduit.

Fresque (ital. affresco). Il y a fresque lorsque les couleurs restent fixées à la paroi par une mince pellicule de carbonate de chaux, produite par le contacte de l'anhydride carbonique de l'air el de la chaux éteinte* contenue :

dans l'enduit humide sur le mur ${ }^{23}$.

mélangée aux couleurs.

Il y a donc deux procédés, dont l'un présente une variante :

a) la chaux est mélangée à l'enduit frais (buon fresco):

b) la chaux est mise au-dessus de l'enduit frais ou sec (dans ce dernier cas. avant de peindre la chaux sèche est mouillée, on parle alors de fresque $a$ secco) ;

c) la chaux est mélangée à la couleur elle-même. elle sert donc de pigment*. Ge procédé est employé surtout pour les retouches finales. quand l'enduit est déjà ser ${ }^{24}$. Ne jas employer le mot fresque comme synonyme de peinture murale* (voir ce mot).

20) Pour tous renseignements complementaires sur des operations de restaumation envisageis. nous nous efforcerons de guider au mieux ceux qui le desireraient.

21 I.es illustralions sont de l'auteur, sauf les fig. 1 el 3 aimablement communiquées par M. H. Kolland, les fig. 5, 9, 10, 11 de .1. J. Deponilly, que je remercie encore do son accuril au musee de soissons et les fig. 6, 12, 1.1, 15 de Mue C. Wilmotte.

22 (irand Larousse Encyclopédique.

23 A. P. IAtric, Frescue dans Encyclopedia Brilannica al iffresco, dans Enciclopedia dell Arle.

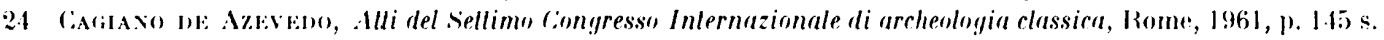


Fresocte mixte (ital. affresco mislo). A la chaux* est mélangé un liant*.

Liant. Tout produit, autre que la claux*, qui permet de lier un mortier*, un enduit*. Par exemple la fresque* pure n'a pas de liant, car c'est la chaux* qui tient ce rôle. En revanche, il y a de nombreux liants pour la tempera* (voir ce mot).

Iокті:к. Le mortier romain est un mélange de sable avec une pâte formée en délayant la chaux dans l'eau ${ }^{25}$. Il y a en général deux couches de mortier (intonaco): la première (ital. rinzaffo) est composée de sable à gros grain et de chaux* ; la seconde (ital. arriccio) est de mème composition mais avec du sable de structure plus fine ${ }^{26}$.

Mortier de tchleav. Vélange de poussière de luileau ou de brique pilée (remplaçant le sable) avec une pâte formée en délayant la chaux* dans l'eau. Ne pas confondre avec le béton de tuileau, où les éléments de tuileaux sont plus gros. La surface de ce mortier*, bien lissée, peut parfois donner l'impression que le mur a été peint en rose ou en rouge. Dans ce cas, la coupe d'un fragment de mortier* ne montre pas de couches différenciées mais une seule épaisseur rosée qui permet de reconnaitre qu'il s'agit d'un mortier de tuileau. Si la coupe révèle une couche supérieure blanche avec pellicule de couleur, il s'agit d'un enduit peint. posé sur ce mortier de tuileau.

Pentere de chevalet. Elle est portative, de format réduit. Son support est en général le bois. Elle peut être incluse dans une peinture murale* (voir ce mot) en guise d'emblema.

Paistere miraif. La peinture murale orne les murs intérieurs ou extérieurs d'un édifice. Elle englobe les techniques comme la fresque ${ }^{*}$. la tempera ${ }^{*}$ la détrempe ${ }^{*}$ ou la peinture à la cire. Le stuc* peint ou coloré ne devrait pas en faire partie, non plus que la mosaïque murale el toutes les techniques où la couleur est incorporée au support comme les badigeons. les crépis.

Pigiment substance colorée, d'origine minérale, organique ou métallique, généralement insoluble. qui colore la surface sur laquelle on l'applique. sans pénétrer dans les fibres ${ }^{27}$. Iin peinture murale antique, seuls les pigments* d'origine minérale ou métallique sont utilisés, à cause du voisinage de la chaux*. excepté le noir, de nature organique, qui a tendance, de ce fait, à pâlir.

STt: (ital. slucco plastico). Le stuc est un revètement extérieur ou intérieur. constitué d'un mélange de chaux* el de poudre de marbre. liés par une colle, qui permet de prolonger le temps de durcissement de la pâte et d'en renforcer la solidité28. La poudre de marbre et la chaux* peuvent être remplacées par du plâtre. Le stuc*, qui est forcément en relief souvent moulé. peut rester blanc, ètre peint ou teinté. Ne pas confondre avec enduit peint* (voir ce mot).

STIC: peint. Les couleurs sont posées sur la surface du stuc et les deux couches distinctes sont visibles en coupe.

STt' coloré ot trintí. La couleur a été mélangée à la pâte du stuc avant son applicalion sur le mur ou sur un moule; en coupe. toute l'épaisseur du stuc est teintiée.

Farx stcc. Ornements en relief. formés de chaux et de sable, comme un mortier*. Lix. : faux sturs* de Zliten ${ }^{23}$.

25) Définilion de P.-.I. 1) val, P'ierres, mortiers ef briques du P'alais des Thermes, dans (iallia, X, 1952. p. 57-61.

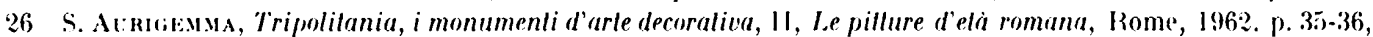
ripport de V. Rosati.

27 Définition du P'elit Robert.

$2 \times$ S. AIRIGEMMa, op. cil., p. 28.

29 Ibid., p. 27 
Trmpera. Les couleurs sont posées sur un enduit* sec gràce à un liant* (voir ce mol). Ces liants sont les colles, les yommes, le lait, l'œuf, elc. (mais pas l'huile).

En dernier lieu, il ne nous semble pas inutile de distinguer deux états des peintures murales, les reconstitutions el les restitutions :

Ri:coxstitction. La reconstilution est l'assemblage effectif de fragments de peinture par recollage et pose - éventuellement -.. sur un support quelconque. Elle est, en général, assez exacte puisque les cassures des fragments s'encastrent les unes dans les autres et qu'il y a peu de lacunes.

Ristitition. Consiste : a) soit en un rapprochement de reconstitutions* partielles avec solution de continuité, en ce cas la différence avec la reconstitution* est l'importance des lacunes;

b) soit en un rapprochement par hypothèse, purement graphique, impliquant la confrontation de divers éléments qui ne s'assemblent pas forcément dans l'ordre proposé.

I.a restitution, susceptible d'erreurs de jugements, est donc moins exacte que la reconstitution.

Catalogee des prodelts chimigles

ACÉTATE DE polyvinyle. Employé en restauration dans une proportion de $10 \%$ avec du caséate de chaux ${ }^{*}$ pour combler les poches d'air situées entre les couches de mortier, supportant une peinture murale.

Casíate de chaux. Préparé par un mélange d'une part de caséine pour trois à sept parts de chaux et d'eau, il sert comme colle et comme fixatif des peintures émincées sur leur nouveau support $^{30}$. Son emploi réclame un climat relativement sec el des fluctuations modérées de température. Il sert également comme composant pour reconstituer le mortier original ou pour remplir les poches d'air.

Chaux Éteinte. Chaux hydratée (hydroxyde $\mathrm{CA}(\mathrm{OH}) 2$ ), poudre blanche, légèrement soluble dans l'eau entrant dans la composition du morlier*.

Charx (lail de). Bouillie de chaux éteinte* délayée dans l'eau, qui sert de pigment blanc pour les rehauts et les délails blane's dans les peintures romaines.

Chat x (eau de). Eau qui contient de la chaux dissoute à saturation $(2 \%)^{31}$; elle a été parfois utilisée comme fixatif de la pellicule picturale mais l'application est longue, irréversible et exige une grande quantité d'eau, c'est pourquoi elle n'est plus conseillée ${ }^{32}$.

Chlortra de polyvinyle. Le chlorure de polyvinyle expansé, comme le polystyrol*, en types commerciaux, est un matériau à cellule fermée, c'est-à-dire imperméable aux gaz et aux liquides ; il est utilisé comme panneau-sandwich dans les restaurations. Il est attaqué par les solvants chlorés et par le méthyléthycétone, résistant aux agents atmosphériques, aux micro-organismes ; les rayons ultra-violets ne l'atteignent pas beaucoup. Ininflammable, il n'est pas sensible à l'humidité. C'est le plus solide des matériaux plastiques expansés, assez facile à façonner après réchauffement. Il faut lui associer des adhésifs aqueux comme les émulsions vinyliques ${ }^{33}$.

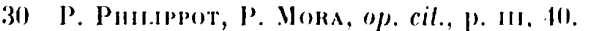

31 Cirand Larousse encyclopédique.

32 P. Pницерот, Р. Мона, ор. cit., p. пा, 22.

33 P. Мora, G. Torraci, op. cit., p. 4.
} 
Cyciofxymanine. Produil utilisé additionné d'eau, comme solvant des matières grasses (repeints i l'huile par exemple), au-dessus de la pellicule picturale. L'eau renforce le pouvoir du solvant: ainsi une solution a $80 \%$ ou $90 \%$ est plus forte qu'une solution à $60 \%$ deau ${ }^{34}$.

Gomme lag̣: C'est une résine qui se présente sous deux formes dans le commerce, en paillettes brunes (à déconseiller en restauration) et en poudre blanche, utilisée en solution de $10 \%$ ¿ $20 \%$ dans de l'alcool éthylique dénaturé, pour fixer la pellicule picturale des peintures murales, ou mème en guise de colle pour détacher des peintures en milieu humide, par exemple les peintures des tombeaux étrusques. L'Institut de la Restauration de Rome recommande de l'employer avec prudence car la gomme laque jaunit et devient insoluble en vieillissant.

Hermétigle: Coxshinass. Employé dans la restauration de monuments dont les pierres ont été désagrégées par les agents atmosphériques. Il remplace les subslances liantes de la pierre devenues pulvérulentes en pénétrant profondément. Il constitue ensuite une couche imperméable a la pluie, une fois le support bien asséché. lin restauration de peinture murale, l'Hermétique Conservans minéralise la mince couche de mortier* restante qui supporte la pellicule picturale. Pour les peintures qu'on désire laisser en place, ce produit sert de fixalif en consolidant l'arriere de la pellicule picturale.

Mowilith. La Mowilith 35/733 sert de colle dans les opérations de restauration. cest un copolymere d'acétale de vinyle et de maléate de dibutyle. elle est soluble dans beaucoup de liquides différents, dont l'acétate d'éthylglycol, solvant employe en restauration. Lille est insoluble dans l'eau el offre une bonne résistance à la lumière et à la chaleur. Dissoute dans les solvants conseillés, elle présente vis-i-vis des pigments* une bonne compatibilité et un fort pouvoir liant.

Paratoï̀ B 72. Méthacrylate d'éthyle en solution à $5 \%$ dans le toluène, ulilisé comme fixalif de la couche picturale apress restauration, de préférence. Il donne satisfaction du point de vue esthétique, présente une forte résistance à la traction, une bonne résistance à l'abrasion et a l'arrachage. ses qualités sont permanentes, il ne jaunit pas. Comme le toluene, il est toxique ${ }^{35}$.

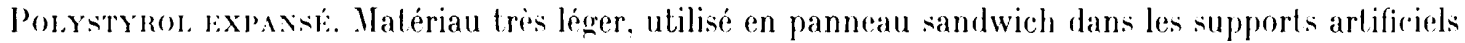
de peinlures murales*. I'n cerlain nombre de ses caractéristiques oblige a prendre avec lui beaucoup de précaulions : il est sensible à l'action des solvants organiques. en particulier aux solvants aromatiques el chlorés, c'est pourquoi il faut toujours le coller au moyen d'adhésifs en émulsion aqueuse ou solubles dans l'eau. Il doit ittre protégé contre les rayons solaires rar les rayons ultra-violets le font jaunir el ses qualités mécaniques dégénèrent. Il est inflammahle ${ }^{36}$.

34 P. PIII.npor, P. Mors, op. ril., p. III, 10.

3i) Ibial., 1. 111, 25-26.

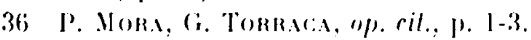

